\title{
Electrically-Assisted Turbocharger Development for Performance and Emissions
}

\author{
Milton Bailey, Leon Tolbert, Norberto Domingo-ORNL, and \\ Brian Bolton, Nabil Hakim - DDC
}

\begin{abstract}
Turbocharger transient lag inherently imposes a tradeoff between a robust engine response to transient load shifts and exhaust emissions. By itself, a wellmatched turbocharger for an engine has limited flexibility in improving this transient response. Electrically-assisted turbocharging has been seen as an attractive option to improve response and lower transient emissions.
\end{abstract}

This paper presents the results of a multi-year joint CRADA between DDC and ORNL. Virtual lab diesel simulation models characterized the performance improvement potential of an electrically assisted turbocharger technology. Operating requirements to reduce transient duration between load shift time by up to $50 \%$ were determined. A turbomachine has been conceptualized with an integrated motor-generator, providing transient burst boost plus energy recovery capability. Numerous electric motor designs were considered, and a prototype motor was developed, fabricated, and is undergoing tests. Power controls have been designed and fabricated. 


\title{
Electrically Assisted Turbo-Charger Development for Performance and Emissions
}

Summary of CRADA \# 1996-2000

Detroit Diesel Corporation, Oak Ridge National Laboratory

\author{
Presented by \\ Houshun Zhang, Detroit Diesel Corporation \\ Milton Bailey, Oak Ridge National Laboratory
}




\section{Outline}

- Motivation

- Transient Engine Operation

- Engine \& Turbocharger Matching

- Transient Engine \& Turbocharger Modeling

- Potential Improvements

- Comparison of Results

- Electric Machinery

- Power Electronics

- Status of Tests 


\section{Motivation}

- Turbo Critical for Power density, Fuel economy, Emission and Driveability.

- Response Time during Transient Operation due to Turbine inertia.

- Improve driveability

- Reduce engine smoke and high PM

- Turbocharger Technology is a Key to Emission Reduction. 


\section{HD FTP Transient Test}
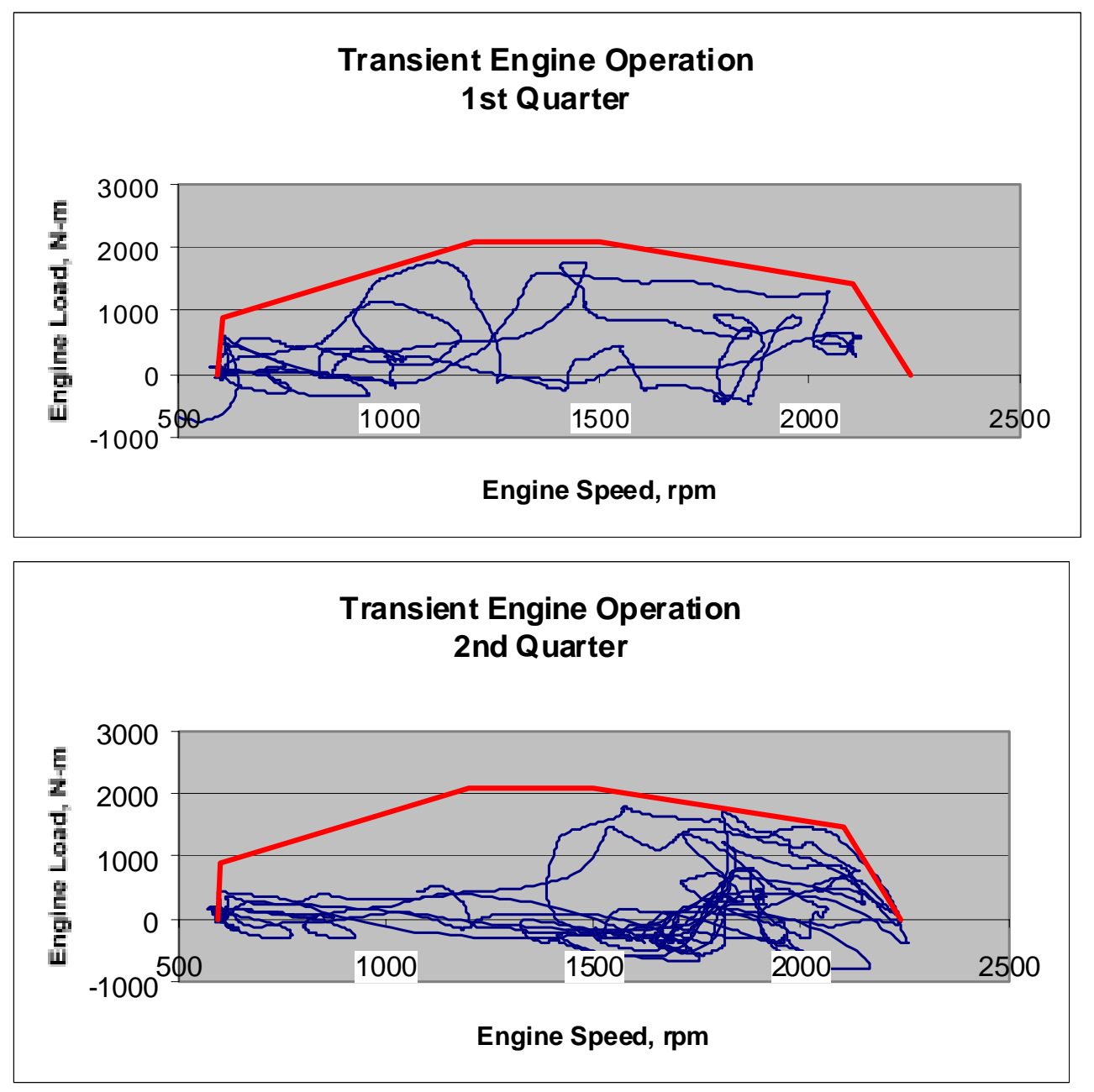

\section{ornl}

999 Detroit Diesel Corporation. All Rights Reserved.

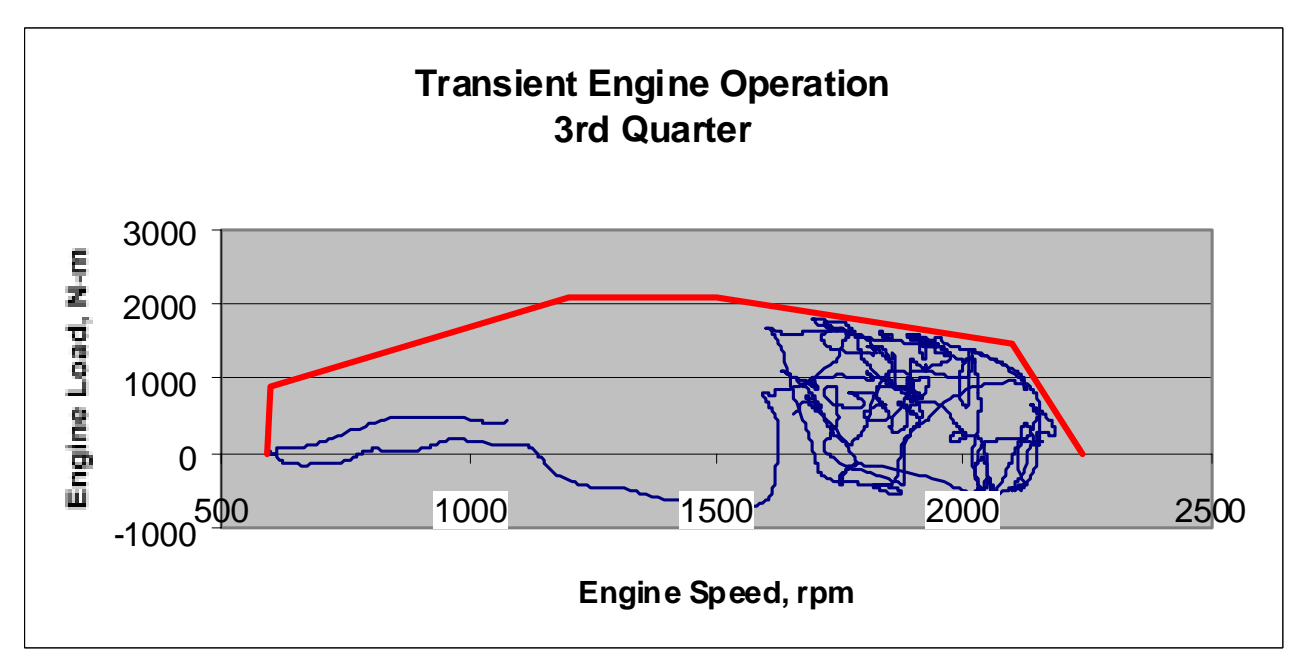

\section{Transient Engine Operation} 4th Quarter

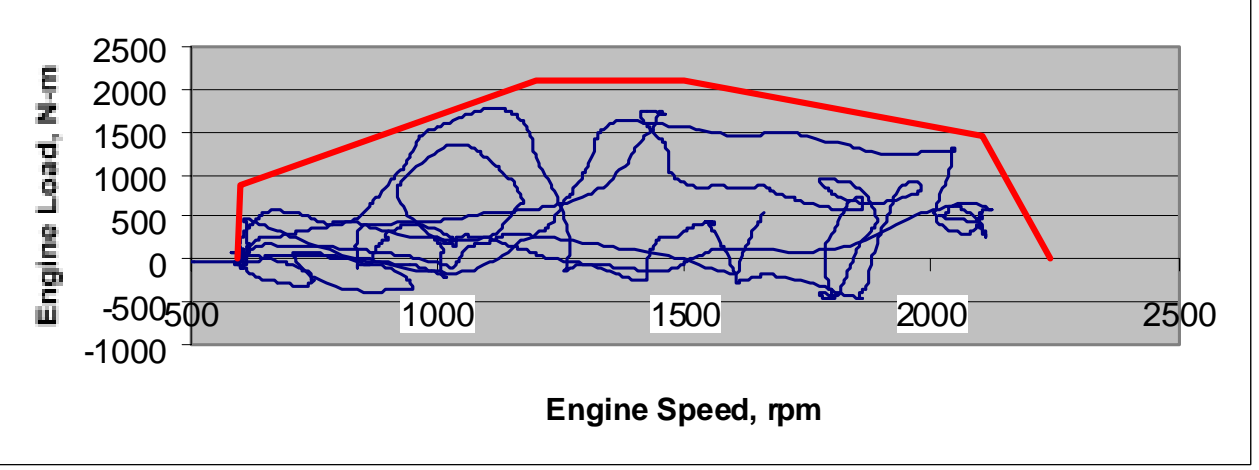




\section{HD FTP Transient Test}

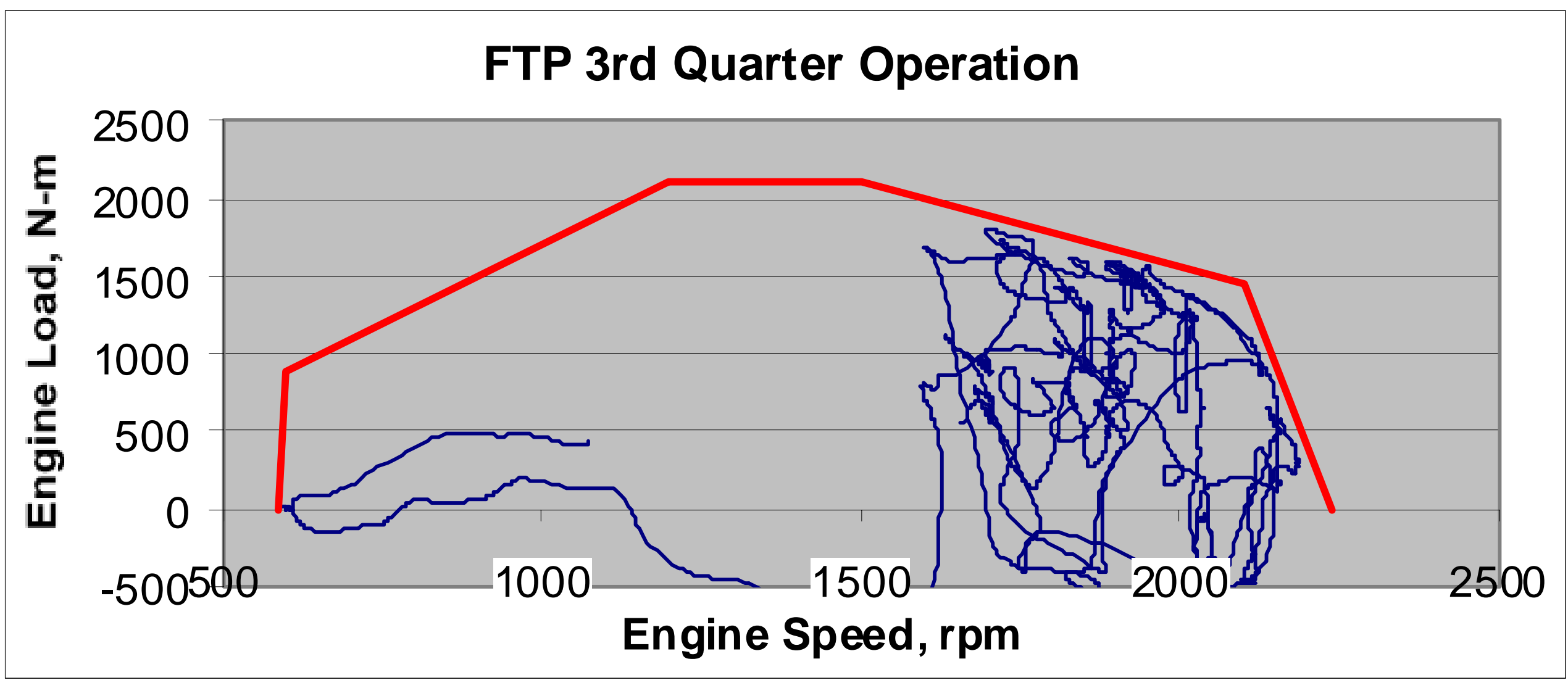




\section{HD FTP Transient Test Turbo Response}

\section{TRANSIENT TURBOCHARGER OPERATION}

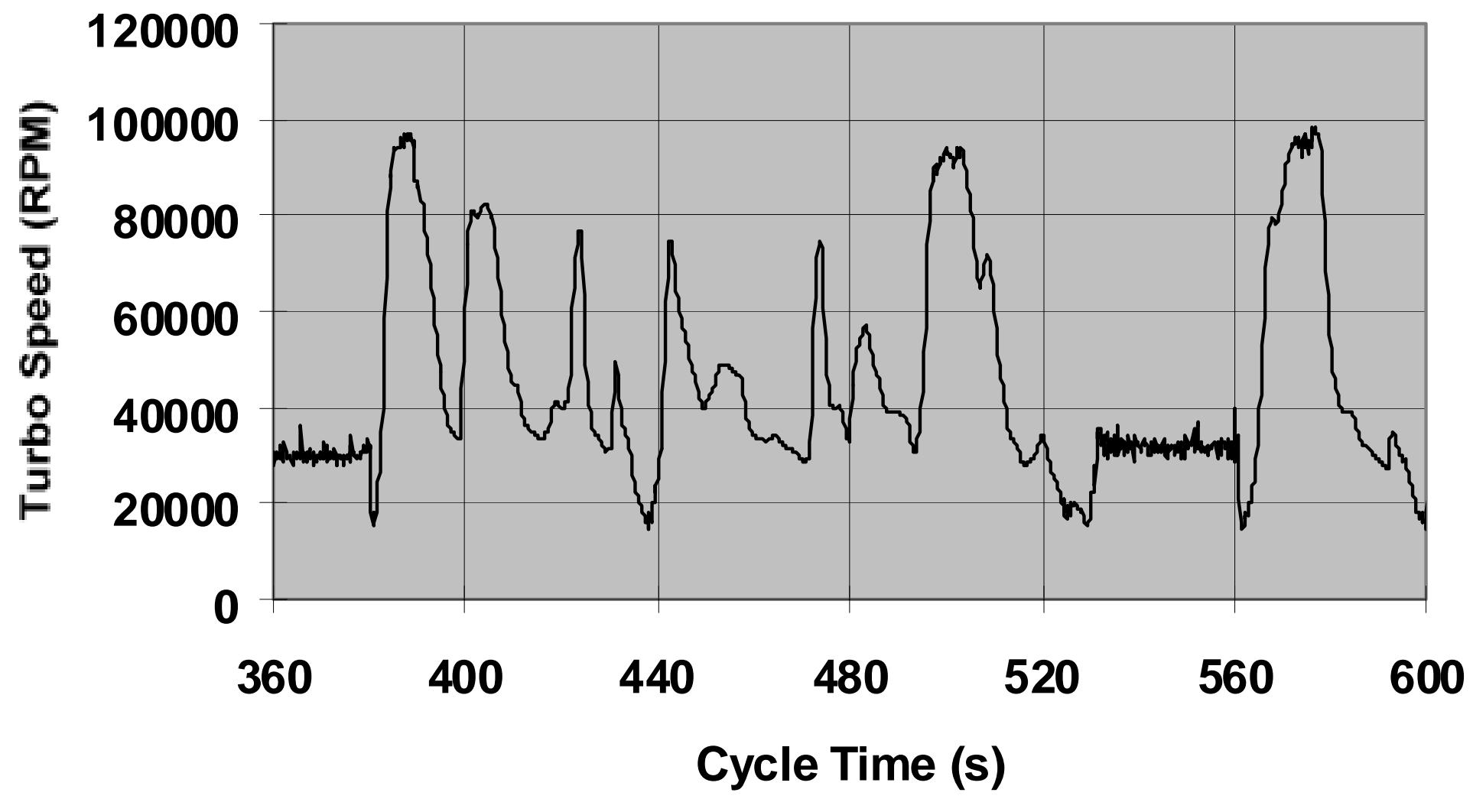




\section{Turbochargers in Emissions Perspective}

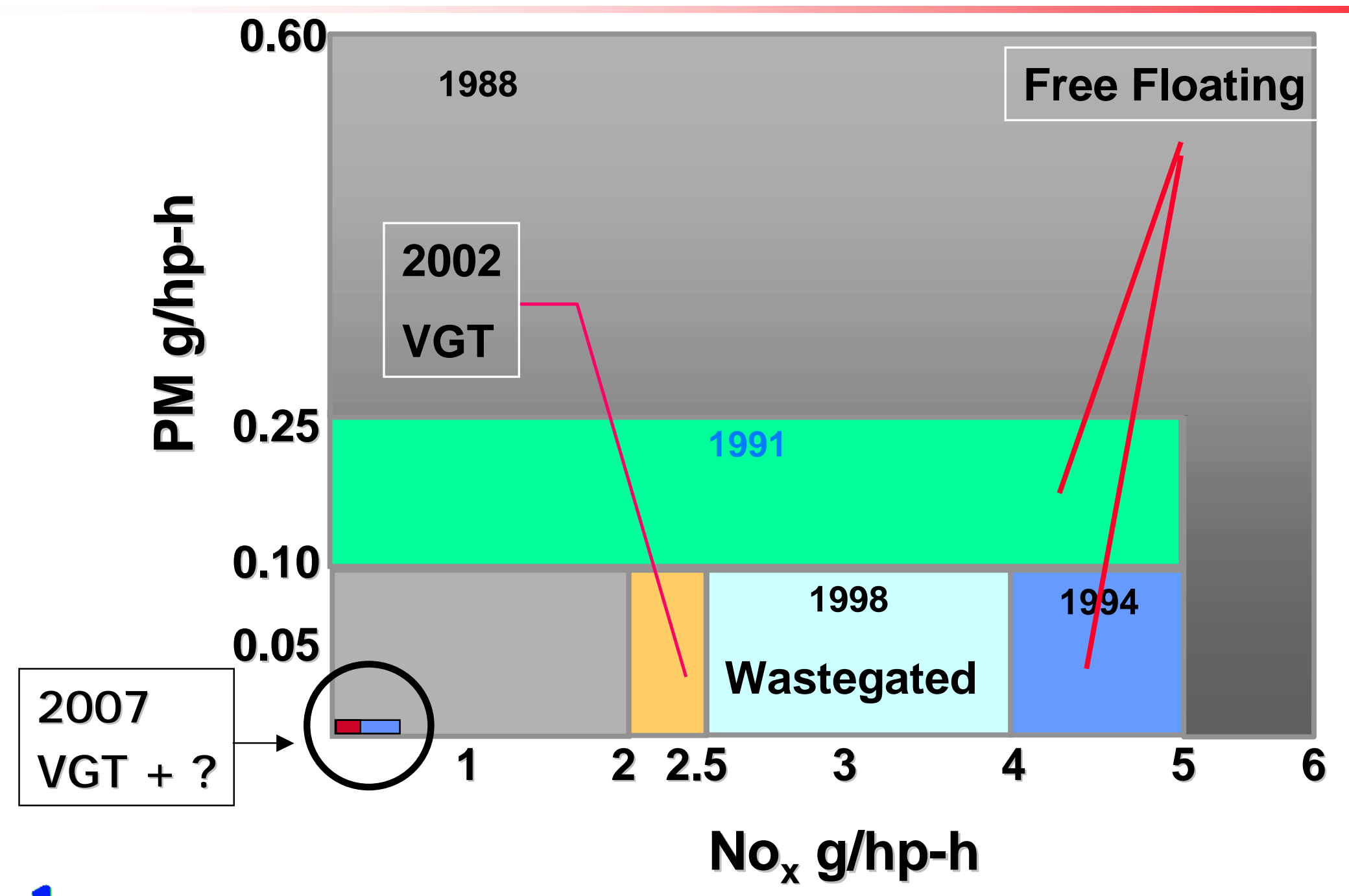




\section{Electrical Turbo Assist}

- Externally drive turbocharger during transient

- Improve emission \& performance at cost of BSFC

- Potentially Use Motor for Steady State BSFC Improvement.

ORNL - 1994 Sketch

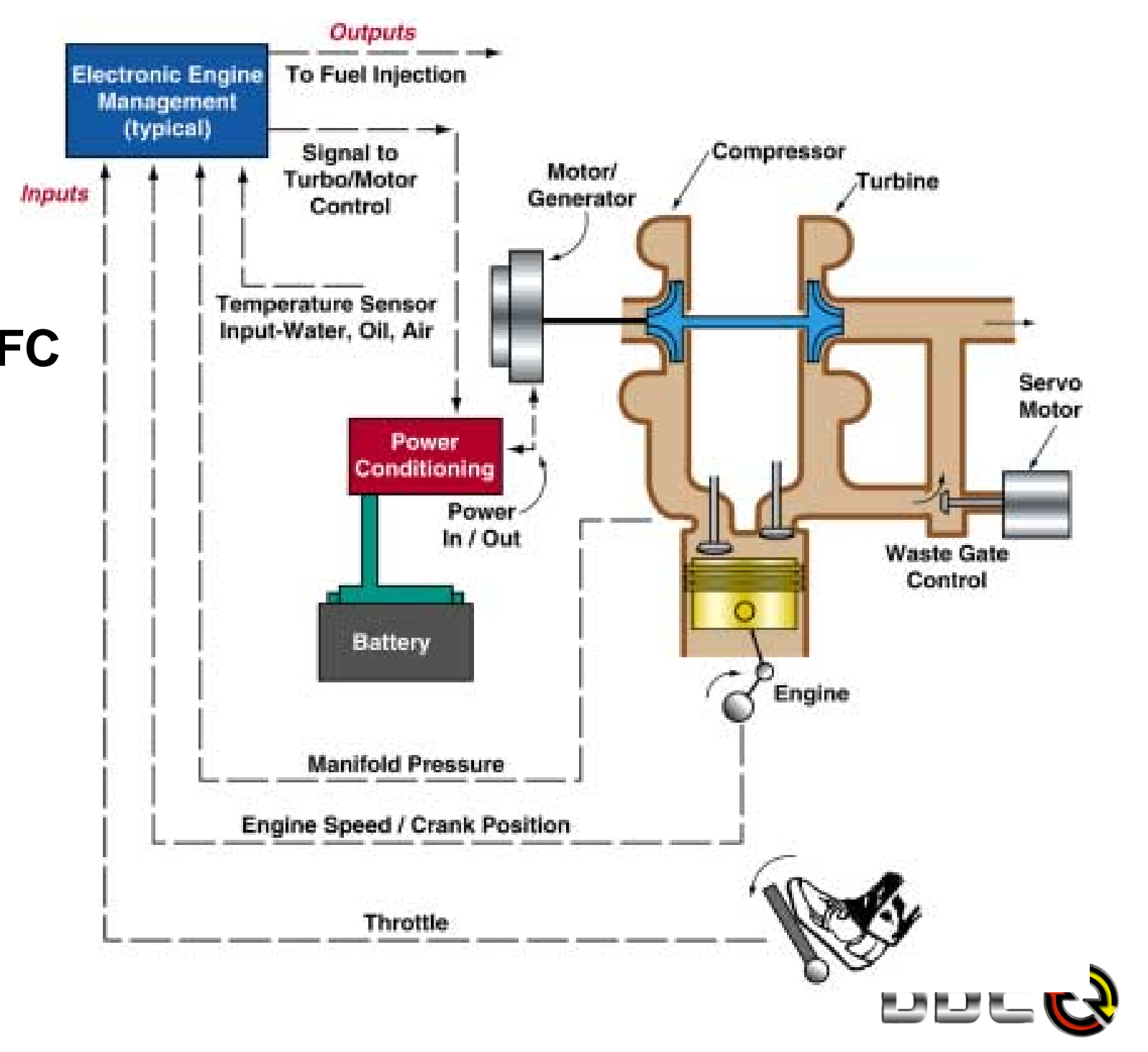




\section{Technical Approach - Virtual Lab}

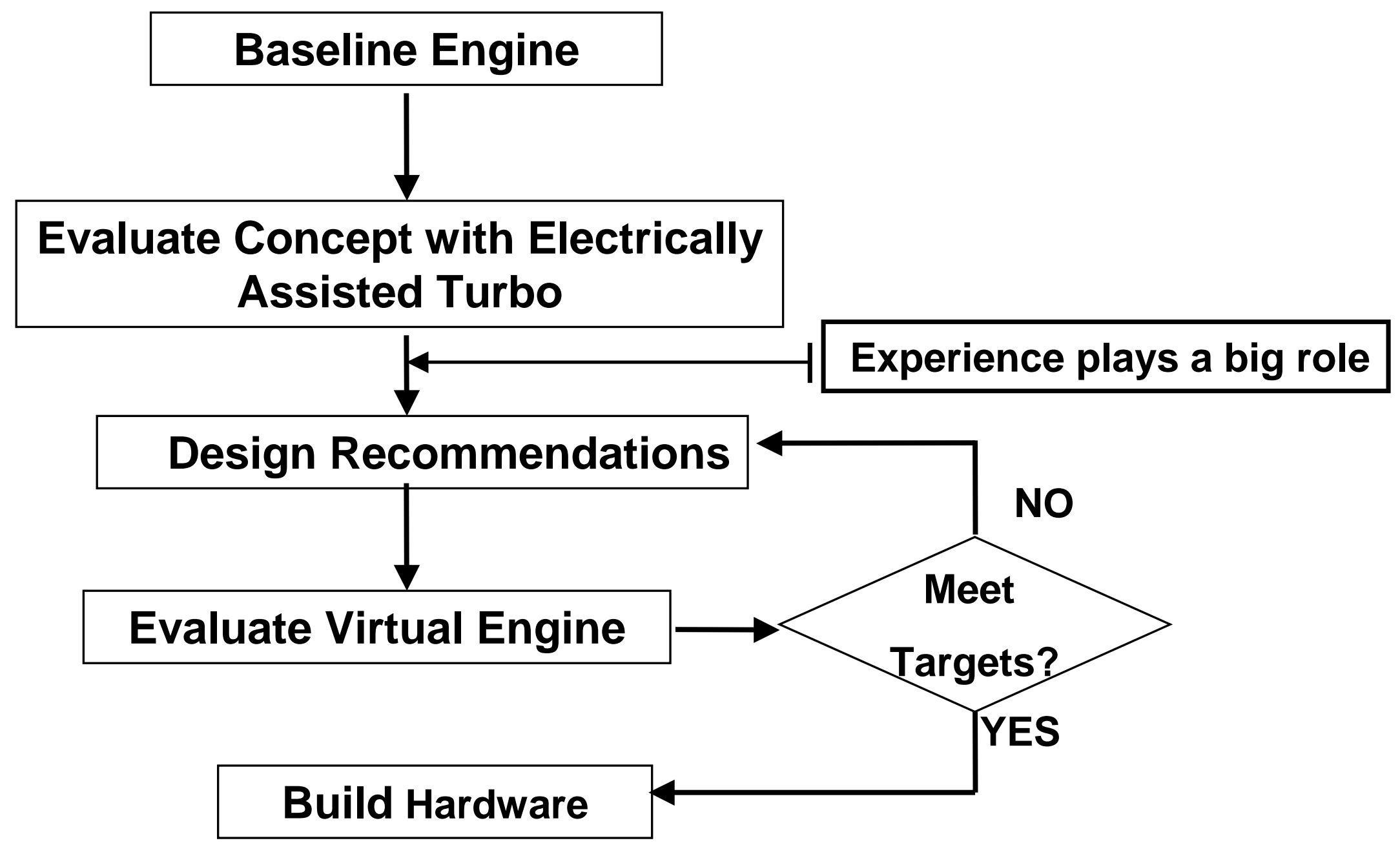




\section{Baseline Engine During Transient Run}

Turbo Speed vs. Time

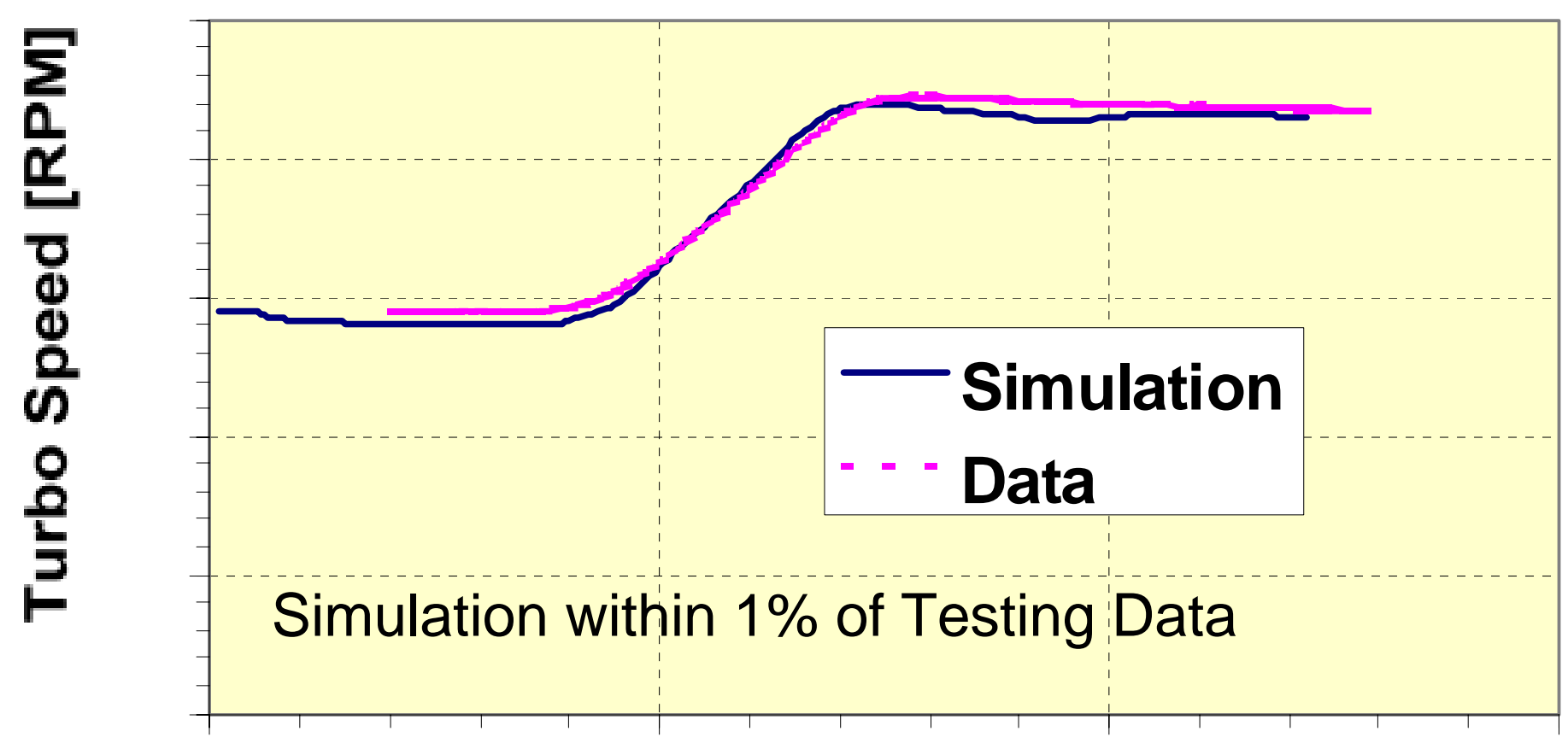

Time [s] 


\section{Simulated Baseline Transient Turbocharger Operation}

- Objective:

- up to $50 \%$ reduction in turbo spool-up time

- What is the Motor Requirement?

\section{Baseline Engine}

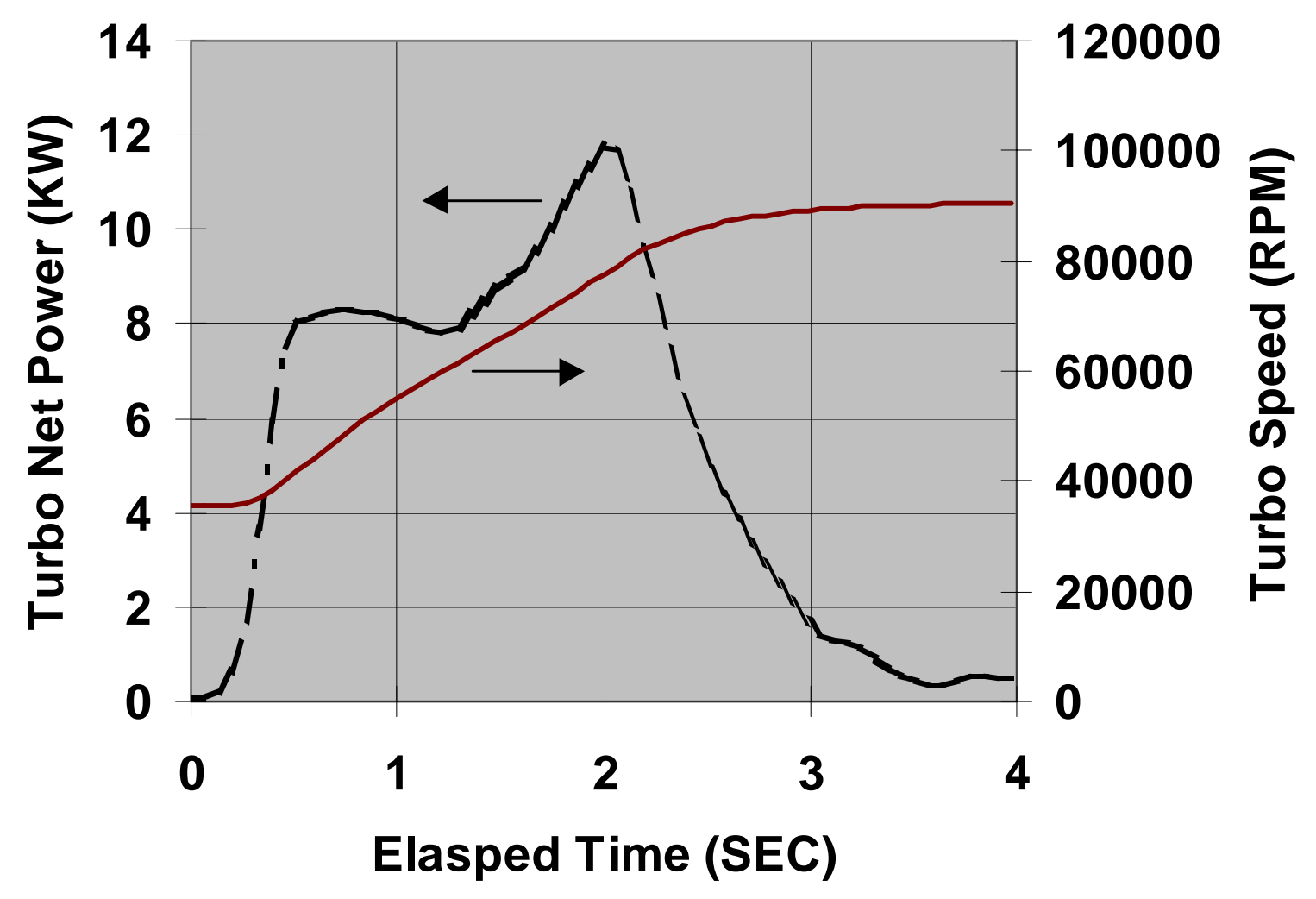




\section{Ramp Acceleration Time for Two Typical Motors}

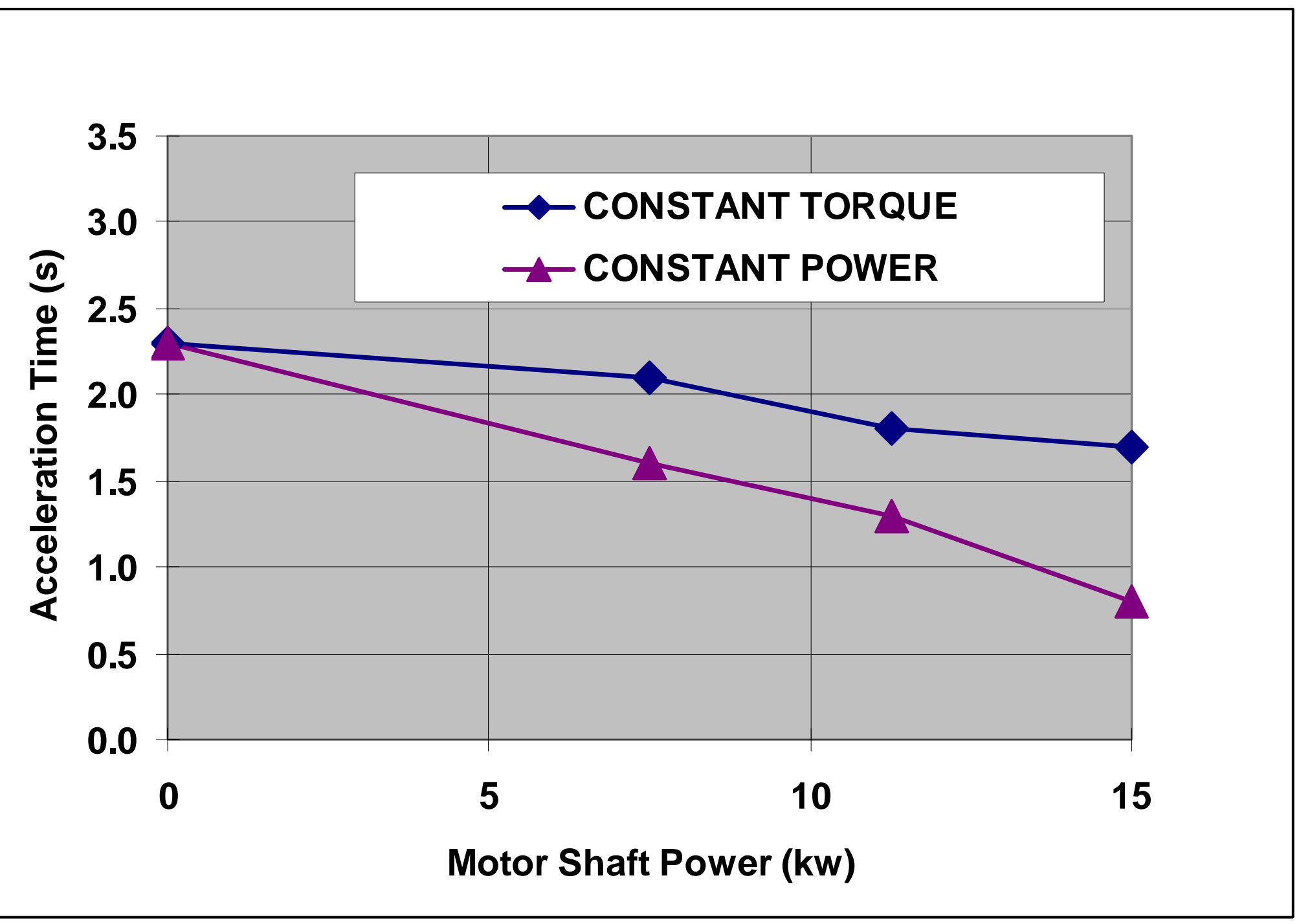




\section{Technology Comparisons Using Virtual Lab}

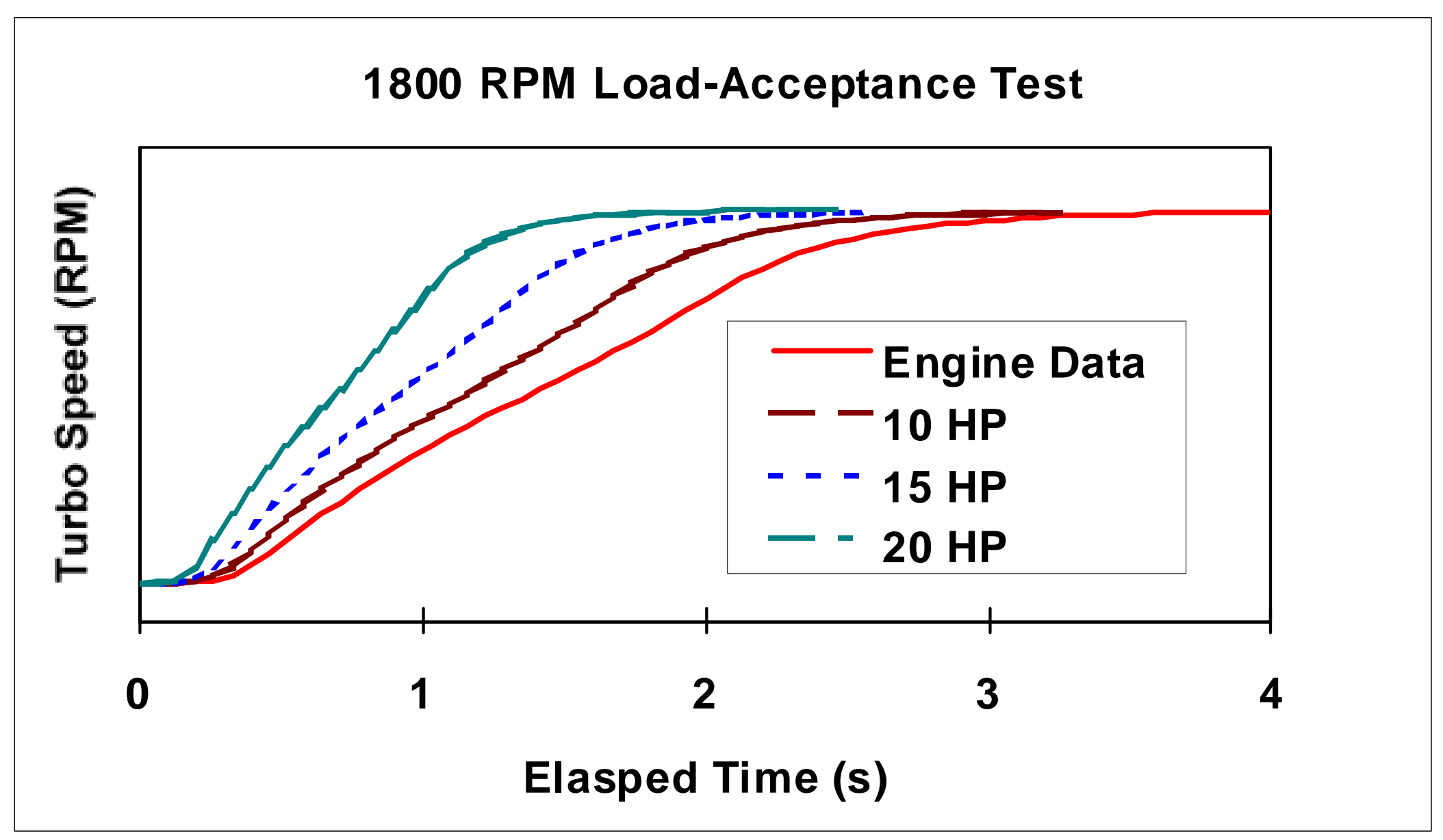

10 HP Power Assist Gives 17\% Reduction of Response 


\section{Electric Motor Design Goals : 10hp constant 20k-100k rpm}

- Motor/alternator options considered included

- Permanent magnet vs switched reluctance

- Axial gap vs radial gap

- Permanent magnet, radial gap, two-pole design selected, 1997.

- High power density and efficiency

- Low polar moment of inertia

- Shaft diameter can be larger

- Facilitates design for high speed

- Disadvantage is larger stator diameter 


\section{Electric motor major components}

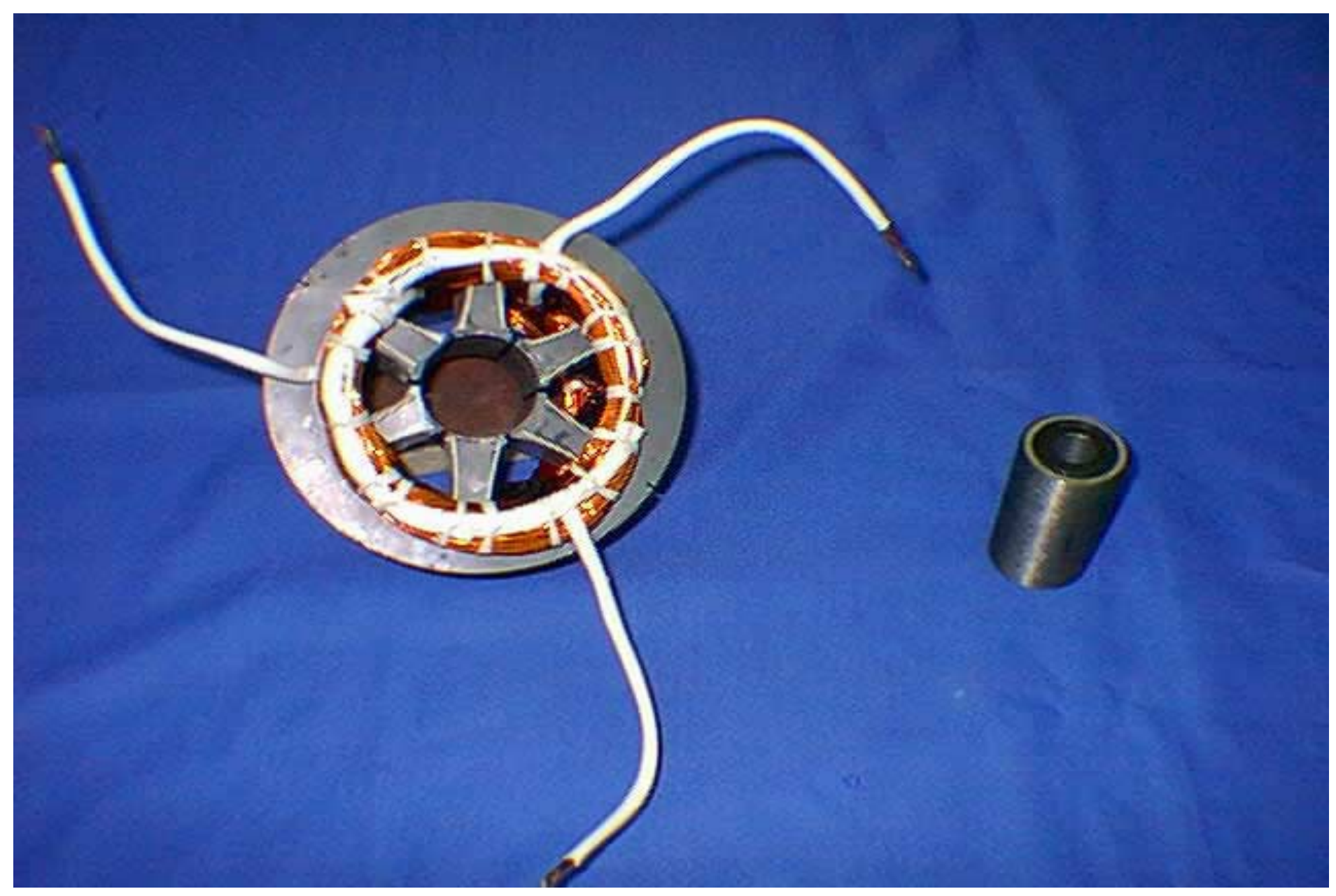




\section{Preliminary motor performance results}

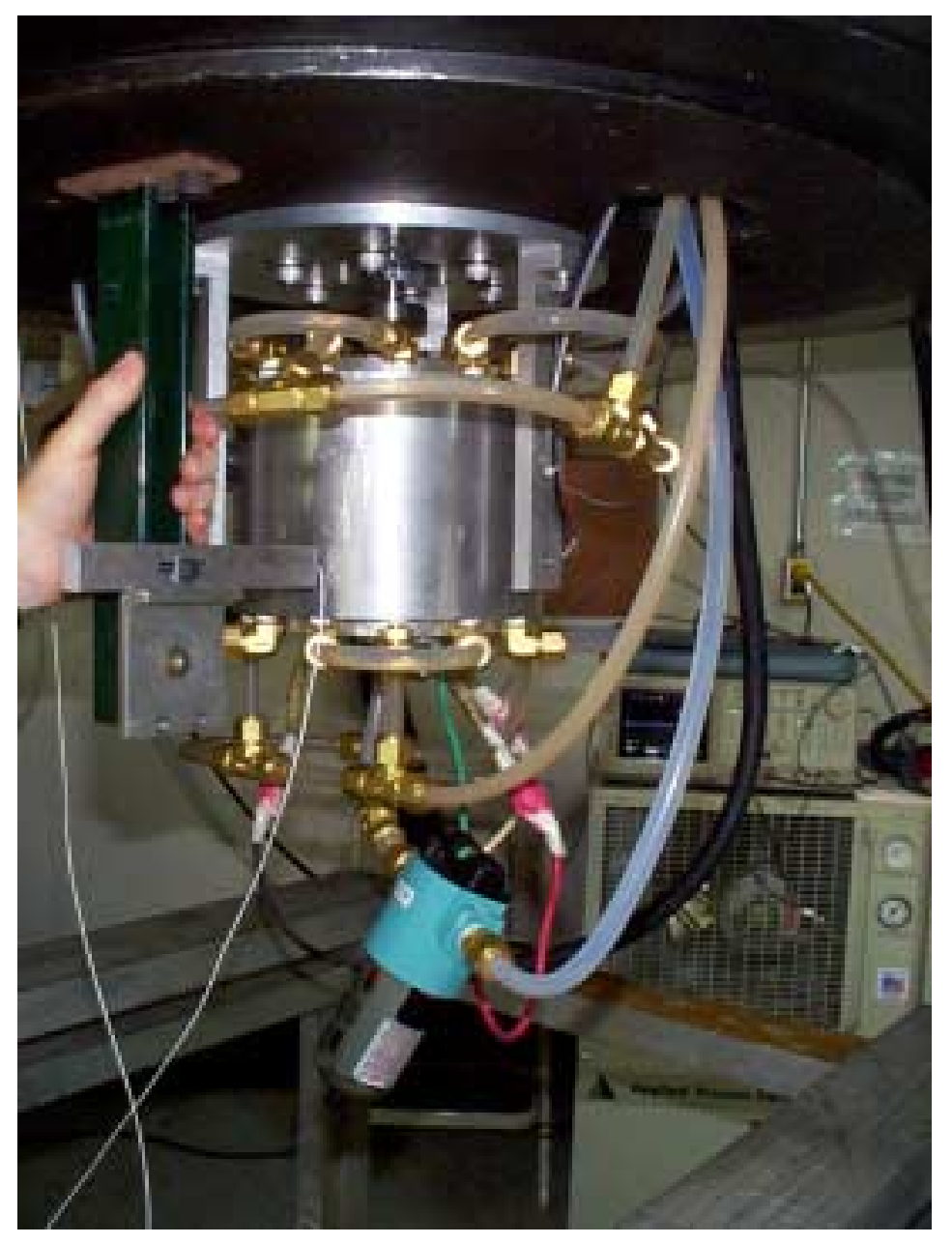

Radial-Gap PM Turbomotor Power Test Data

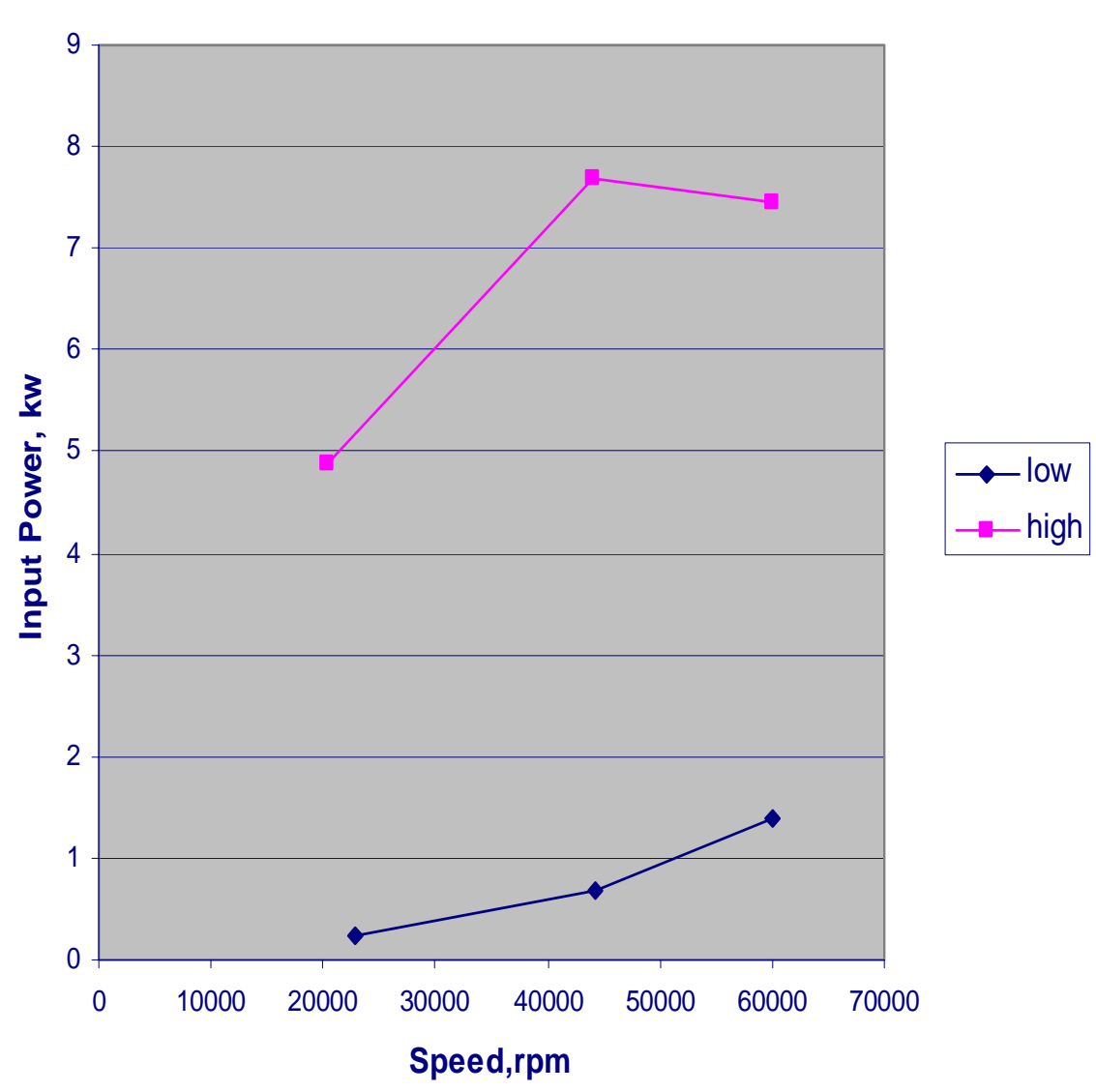




\section{Concluding Remarks}

- Electric Assisted Turbo is One of Promising Technologies to Achieve Fast Response.

- Motor \& sensorless controller conceived, designed and developed.

- Demonstration hardware has been built and early validation achieved.

- Design enhancements have been identified which should lead to a more robust design. 


\section{Acknowledgements}

- ORNL additional team members - Norberto Domingo, Leon Tolbert, Wayland Blake, Bill Allington, Gui-Jia Su

- Detroit Diesel Corporation additional team members Tim Prochnau, Craig Savonen, Nabil Hakim

- Sponsor - DOE/NE, Dr. Bill VanDyke 